\title{
Prediction of spontaneous autoimmune diabetes in NOD mice by quantification of autoreactive $T$ cells in peripheral blood
}

\author{
Jacqueline D. Trudeau, ${ }^{1,2}$ Carolyn Kelly-Smith, ${ }^{1}$ C. Bruce Verchere, ${ }^{1}$ John F. Elliott, ${ }^{3}$ \\ Jan P. Dutz, ${ }^{4}$ Diane T. Finegood, ${ }^{2}$ Pere Santamaria, ${ }^{5}$ and Rusung Tan ${ }^{1}$

\begin{abstract}
${ }^{1}$ Department of Pathology and Laboratory Medicine, University of British Columbia and British Columbia's Children's Hospital, Vancouver, British Columbia, Canada

${ }^{2}$ Diabetes Research Laboratory, School of Kinesiology, Simon Fraser University, Burnaby, British Columbia, Canada

${ }^{3}$ Department of Medical Microbiology and Immunology, University of Alberta, Edmonton, Alberta, Canada

${ }^{4}$ Department of Medicine, University of British Columbia and British Columbia's Children's Hospital, Vancouver,

British Columbia, Canada

${ }^{5}$ Department of Microbiology and Infectious Diseases, and Julia McFarlane Diabetes Research Centre, Faculty of Medicine, University of Calgary, Calgary, Alberta, Canada
\end{abstract}

\begin{abstract}
Autoimmune (type 1) diabetes mellitus results from the destruction of insulin-producing pancreatic $\beta$ cells by $T$ lymphocytes. Prediction of cell-mediated autoimmune diseases by direct detection of autoreactive $T$ cells in peripheral blood has proved elusive, in part because of their low frequency and reduced avidity for peptide MHC ligands. We demonstrate here that MHC class I tetramers complexed to a high-avidity analogue of an immunodominant $\beta$ cell epitope detect diabetogenic $\mathrm{CD}^{+} \mathrm{T}$ cells in the peripheral blood of NOD mice ex vivo and that the quantification of this autoreactive $T$ cell population in peripheral blood is a powerful predictor of autoimmune diabetes.

This article was published online in advance of the print edition. The date of publication is available

from the JCI website, http://www.jci.org. J. Clin. Invest. 111:217-223 (2003). doi:10.1172/JCI200316409.
\end{abstract}

\section{Introduction}

Type 1 diabetes is an autoimmune disorder characterized by the selective destruction of pancreatic $\beta$ cells, resulting in insulin deficiency and hyperglycemia. Studies of both humans (1) and the NOD mouse model $(2,3)$ have shown that $\beta$ cell destruction is mediated largely by $\mathrm{T}$ lymphocytes. Despite the direct $\beta$ cell cytotoxic role of T cells during diabetes progression, prediction of disease in both humans and the NOD mouse has been based primarily on the presence of circulating autoantibodies to putative $T$ cell antigens $(4,5)$. Detection and characterization of antigen-specific $T$ cells from peripheral blood during the progression of type 1 diabetes, or other autoimmune diseases, has thus far not been possible without in vitro manipulation. Consequently, little is known regarding the evolution of

Received for publication July 12, 2002, and accepted in revised form October 29, 2002

Address correspondence to: Rusung Tan, Department of Pathology and Laboratory Medicine, British Columbia's Children's Hospital, 4480 Oak Street, Vancouver, British Columbia V6H 3V4, Canada. Phone: (604) 875-3605; Fax: (604) 875-3777; E-mail: roo@interchange.ubc.ca. Conflict of interest: The authors have declared that no conflict of interest exists.

Nonstandard abbreviations used: insulin B chain (INS); enzymelinked immunospot (ELISpot); $\mathrm{T}$ cell receptor-transgenic (TCR-transgenic); glutamic acid decarboxylase (GAD); insulin autoantibodies (IAA); human leukocyte antigen (HLA). autoreactive $\mathrm{T}$ cell populations during the natural history of autoimmune diseases.

Although the detection of antigen-specific $\mathrm{T}$ cells using MHC tetramers has permitted careful characterization of $\mathrm{T}$ cell responses to many viral and bacterial infections $(6,7)$, attempts to elucidate the in vivo dynamics of autoreactive $\mathrm{T}$ cells during the progression of autoimmune diseases have been less successful. With a single exception (8), attempts to visualize autoreactive T cells in peripheral blood or lymphoid organs ex vivo with MHC tetramers bearing naturally-occurring ligands have failed (9-14), possibly because of the lower avidity that $\mathrm{T}$ cells have for self versus foreign peptide/MHC ligands $(15,16)$. To overcome this limitation, we have engineered high-avidity peptide/MHC tetramers capable of stably interacting with low-avidity autoreactive $T$ cells, thus facilitating their detection. The visualization of lowavidity $T$ cell populations in peripheral blood would provide a simple, minimally invasive method for assessing the presence of autoreactive $T$ cells within infiltrated tissues and might therefore be useful for predicting the development of autoimmune disease.

The feasibility of employing a high-avidity MHC tetramer to detect relatively low-avidity autoreactive $\mathrm{T}$ cells was investigated using NOD mice, an extensively studied model of human type 1 diabetes $(3,17)$. In these mice, diabetes develops by 16 to 20 weeks of age in approximately $80 \%$ of females and is preceded by a prolonged period of mononuclear cell inflammation of the 
pancreatic islets (insulitis) that begins at approximately 3-4 weeks of age. During the period spanning the progression of insulitis to overt diabetes, autoreactive $T$ cell populations expand, accumulate, and destroy $\beta$ cells.

$\mathrm{CD}^{+}$CTLs are an essential component of $\beta$ cell destruction and are necessary for diabetes development $(18,19)$. To date, two $\beta$ cell-specific CTL epitopes have been described in NOD mice. The first is an epitope derived from the insulin B chain (INS) (9), and the second is a peptide mimotope designated NRP (20), for which the endogenous counterpart is currently unknown. $\mathrm{CD}^{+}$ $T$ cells specific for INS have been reported to account for the majority of $\mathrm{T}$ cells within the pancreatic islets of young NOD mice (9). In contrast, NRP-reactive $\mathrm{CD}^{+} \mathrm{T}$ cells predominate in cultured pancreatic islets from NOD mice in the weeks just before the onset of diabetes (10). As well, $\mathrm{T}$ cell receptor-transgenic (TCR-transgenic) NOD mice that express an NRP-reactive TCR (8.3-NOD mice) develop diabetes at an accelerated rate, suggesting that these particular CTLs are important mediators of $\beta$ cell damage in vivo (21). In this study, high-avidity peptide/MHC class I tetramers were developed and used to determine the temporal relationship between the appearance of autoreactive $T$ cells in peripheral blood, secondary lymphoid organs, and pancreatic islets and the development of diabetes. We show, we believe for the first time, that the development of an autoimmune disease can be predicted based on the presence of antigen-specific cytotoxic $\mathrm{T}$ cells in the peripheral blood.

\section{Methods}

Mice. Female NOD mice were purchased from The Jackson Laboratories (Bar Harbor, Maine, USA) and maintained in a specific pathogen-free animal facility at our institution. Blood glucose was monitored using a Glucometre Elite monitor (Bayer Canada, Toronto, Ontario, Canada), and diabetes was defined as two consecutive readings $\geq 15 \mathrm{mM}$. Diabetic mice were maintained on $0.5 \mathrm{U} /$ day of Humulin NPH (Eli Lilly, Toronto, Ontario, Canada) or with LinBit insulin implants (LinShin Canada Inc., Scarborough, Ontario, Canada). All animal experiments were performed in accordance with the rules of the Animal Care Committee, University of British Columbia.

Peptides and tetramers. The peptides NRP (KYNKANWFL), NRP-A7 (KYNKANAFL), NRP-V7 (KYNKANVFL), INS (LYLVCGERL), and TUM (KYQAVTTTL) were prepared by FMOC chemistry and purified by reverse-phase HPLC (> 90\% purity) at the University of British Columbia. The INS peptide was modified (Gly9Leu) from its endogenous counterpart to increase MHC class I stability, without affecting CTL binding ability. $\mathrm{H} 2-\mathrm{K}^{\mathrm{d}}$ tetramers were prepared as described (22) and conjugated to streptavidin-phycoerythrin (Rockland, Gilbertsville, Pennsylvania, USA). Tetramer function was validated by staining spleen cells from 8.3-TCR NOD mice or the G9C8 CD8 ${ }^{+} \mathrm{T}$ cell clone (gift of F. Susan Wong).

Islet isolation and FACS staining. Pancreatic islets were isolated by collagenase perfusion (type V; Sigma-Aldrich,
Oakville, Ontario, Canada) of the common bile duct and separated on a dextran gradient. Islets were dispersed into single cells to liberate lymphocytes by incubation with Cell Dissociation Buffer (Life Technologies Inc., Burlington, Ontario, Canada). Single cell suspensions from islets, peripheral blood, spleen, and pancreatic draining lymph nodes were stained with tetramer for 3 hours, then with FITC-conjugated anti-CD8 (clone YTS 169.4; Cedarlane Laboratories Ltd., Hornby, Ontario, Canada) and PerCP-conjugated anti-B220 (clone RA3-6B2; PharMingen, San Diego, California, USA) for 30 minutes, all on ice. For animals younger than 8 weeks of age, islets were pooled from several animals to obtain sufficient cells for analysis. Stained cells were analyzed by flow cytometry (FACSCalibur; Becton Dickinson Immunocytometry Systems, San Diego, California, USA). Tetramer positivity was determined using a lymphocyte gate and exclusion of $\mathrm{B} 220^{+}$cells. Tetramer-positive cells are expressed throughout as percentage of $\mathrm{CD}^{+} \mathrm{B}^{-} 20^{-}$cells, minus the percentage of TUM tetramer-positive cells (< $0.05 \%$ for peripheral blood analysis). Data was analyzed using FCSpress software (FCSPress, Cambridge, United Kingdom).

Peripheral blood. Blood (130 $\mu \mathrm{l})$ was collected from the saphenous vein using heparinized capillary tubes (Fisher Scientific Ltd., Nepean, Ontario, Canada) and placed in lysis buffer $\left(0.15 \mathrm{M} \mathrm{NH}_{4} \mathrm{Cl}, 1.0 \mathrm{mM} \mathrm{KHCO}_{3}, 0.1 \mathrm{mM}\right.$ $\mathrm{Na}_{2}$ EDTA) to remove red blood cells prior to staining. Enzyme-linked immunospot assays. Enzyme-linked immunospot (ELISpot) plates (Millipore Corp., Bedford, Massachusetts, USA) were precoated with IFN- $\gamma \mathrm{Ab}$ (clone R4-6A2; PharMingen) and blocked with complete medium containing FCS. Dispersed islet cells were coincubated with $5 \times 10^{5}$ P815 cells (American Type Culture Collection, Manassas, Virginia, USA) and NRP-V7, INS, or TUM peptides $(1 \mu \mathrm{g} / \mathrm{ml})$ for 36 hours. IFN- $\gamma$ secretion was detected with a second, biotinylated IFN- $\gamma \mathrm{Ab}$ (clone XMG1.2; PharMingen). Spots were developed using alkaline phosphatase conjugate substrate buffer (Bio-Rad Laboratories Inc., Hercules, California, USA), counted, and expressed as a proportion of the total number of islet cells.

\section{Results}

Detection of autoreactive $C D 8^{+} T$ cells. To establish first that NRP-reactive $\mathrm{CD}^{+} \mathrm{T}$ cells could be detected directly ex vivo, $\mathrm{H} 2-\mathrm{K}^{\mathrm{d}}$ tetramers were generated bearing the peptides NRP, NRP-A7, and NRP-V7. NRP-V7 is a heteroclitic analogue of the previously described peptides NRP and NRP-A7, possessing superior agonistic activity $(10,23)$. $\mathrm{H} 2-\mathrm{K}^{\mathrm{d}}$ tetramers bearing the $\beta$ cell peptide, INS, and an irrelevant peptide, TUM (24), were used as controls. MHC class I tetramers complexed to NRP-V7 stained islet-associated $\mathrm{T}$ cells more intensely and bound to a significantly greater number of $\mathrm{T}$ cells than tetramers bearing the peptides NRP or NRP-A7 (Figure 1a). We therefore chose to examine freshly isolated islets using the index peptide, NRP, the high-avidity analogue NRP-V7, as well as the previously reported INS peptide (Figure 1, b and c, and 

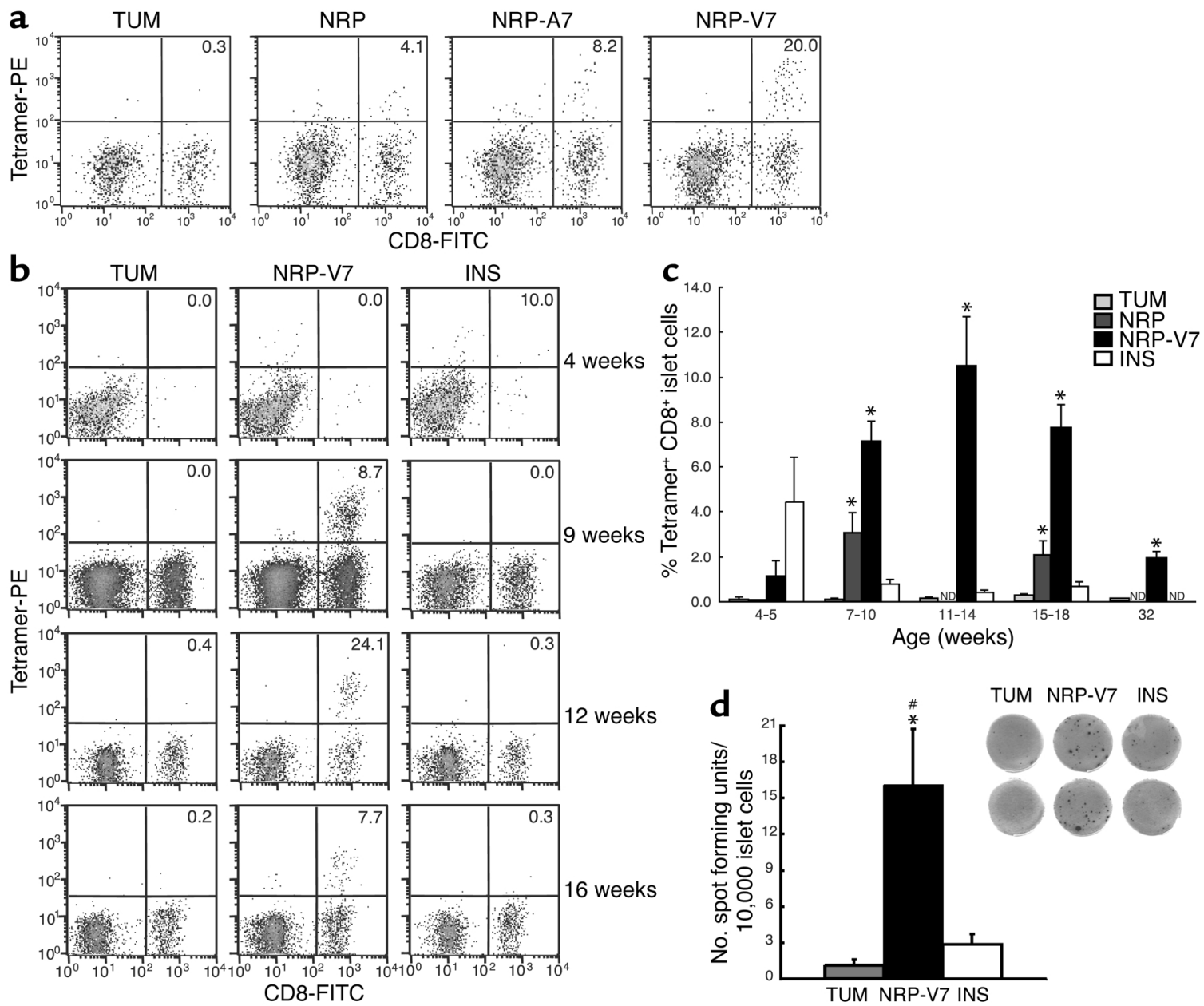

Figure 1

High-avidity peptide/MHC class I tetramers detect a higher frequency of autoreactive T cells from freshly isolated islets. All numbers indicate percentage of $\mathrm{CD}^{+}{ }^{+}$B220- tetramer $^{+}$cells. (a) Pancreatic islets derived from 8-week-old female NOD mice were stained with tetramers associated with the indicated peptides. Data are representative of six independent experiments from mice 6-15 weeks of age. Similar results (but approximately tenfold lower) were observed with peripheral blood (data not shown). (b) Representative examples of TUM, NRP-V7, and INS tetramer staining of islets at 4, 9, 12, and 16 weeks of age. (c) Mean percentage ( \pm SEM) of tetramer-positive cells from mice at 4-5 $(n=4), 7-10(n=14), 11-14(n=17)$, and 15-18 $(n=14)$ weeks of age. NRP staining performed only at 4-5 weeks, $n=3$; 7-10 weeks, $n=7 ; 15-18$ weeks, $n=10$. Data is shown also for nondiabetic mice at 32 weeks of age $(n=5)$. ND, not determined; PE, phycoerythrin. ${ }^{*} P<0.001$ compared with TUM at each age. (d) Islet cells pooled from 4 mice (13-16 weeks of age) were assayed by ELISpot for IFN- $\gamma$ secretion in response to TUM, NRP-V7, or INS $(n=4)$. Naive spleen cells from an 8.3-TCR NOD mouse were used as negative control. ${ }^{*} P<0.0001$ compared with TUM, ${ }^{*} P<0.05$ compared with INS. Inset: representative ELISpot assay: duplicate wells containing 20,000 islet cells from 14-week-old NOD mice.

Table 1). In freshly isolated islets from 4- to 5-week-old mice, the number of INS-reactive $\mathrm{CD}^{+} \mathrm{T}$ cells ranged from $0.60 \%$ to $10.0 \%$, a value less than that reported by others (9). The proportion of INS-reactive cells in the islets declined to less than $3 \%$ by $7-10$ weeks of age and declined further thereafter. In contrast, the NRP-reactive population of $\mathrm{CD}^{+} \mathrm{T}$ cells first appeared at 7 weeks of age and peaked at 11-14 weeks of age, when the proportion of $\mathrm{CD}^{+}$cells within islets that stained with the NRPV7 tetramer ranged from $1.61 \%-36.8 \%$. Although the age of peak NRP-V7 staining roughly paralleled that of NRP, use of the high-avidity NRP-V7 analogue permitted detection of a much higher number of autoreactive $T$ cells as compared with the index NRP mimotope (Figure $1 \mathrm{c}$ and Table 1). To confirm that the NRP-V7-reactive cells were functional, we counted the number of isletassociated cells that produced IFN- $\gamma$ in response to NRPV7, INS, or TUM peptides using ELISpot assays (Figure 1d). Approximately $0.2 \%$ of total islet cells secreted IFN- $\gamma$ in response to NRP-V7-a number that was consistent with the proportion of NRP-V7-reactive T cells contained within the total islet cell population as determined by NRP-V7 tetramer staining and a value significantly greater than that obtained using either INS or TUM.

Detection of peripheral blood autoreactive T cells. Having established that INS and NRP-reactive T cells could be detected from islets ex vivo, we attempted next to determine whether they were detectable in peripheral blood and if their presence could act as a surrogate marker for infiltration of pancreatic islets. Although INS-reactive 
Table 1

Range of autoreactive $\mathrm{CD}^{+} \mathrm{T}$ cells in freshly isolated pancreatic islets

$\begin{array}{lccc}\text { Age (weeks) } & \begin{array}{c}\mathrm{NRP}-\mathrm{V} 7^{+} \mathrm{CD} 8^{+} \\ \text {cells }(\%)\end{array} & \begin{array}{c}\mathrm{NRP}^{+} \mathrm{CD} 8^{+} \\ \text {cells }(\%)\end{array} & \begin{array}{c}\mathrm{INS}^{+} \mathrm{CD} 8^{+} \\ \text {cells }(\%)\end{array} \\ 4-5 & 0.0-2.68 & 0.0 & 0.60-10.0 \\ 7-10 & 0.32-14.1 & 1.01-7.10 & 0.0-2.48 \\ 11-14 & 1.61-36.8 & \text { Not determined } & 0.0-1.37 \\ 15-18 & 1.79-18.7 & 0.25-7.13 & 0.0-1.52 \\ 32 & 1.18-3.01 & \text { Not determined } & \text { Not determined }\end{array}$

$\mathrm{CD}^{+} \mathrm{T}$ cells were detectable in the islets of 4- to 5-weekold mice (Figure 1, b and c), at all time points examined (weekly intervals from 4 to 18 weeks of age), INS-reactive T cells could not be detected in the peripheral blood. The NRP-V7 tetramer was chosen to analyze the peripheral blood of NOD mice, because in a fashion similar to the pancreatic islets (Figure 1a), NRP-V7 tetramers detected a higher proportion of NRP-reactive cells in the peripheral blood than the NRP-A7 or NRP tetramers (data not shown). Mice from three age-specific groups representing early (9-10 weeks), middle (11-14 weeks), and late (15-17 weeks) stages of disease pathogenesis were sacrificed, and blood, islets, and lymphoid organs from each mouse were stained directly ex vivo with the NRP-V7 tetramer. Autoreactive T cells were clearly detectable in peripheral blood from each group of mice, and their presence in circulation was associated with the presence of autoreactive $\mathrm{T}$ cell infiltration of both pancreatic islets and lymphoid organs (Figure 2). In the vast majority of the animals examined (27/30), the presence of NRP-V7reactive $T$ cells in peripheral blood correlated with the presence of a similar population of $\mathrm{T}$ cells in pancreatic islets; however, the exact percentage of NRP- $7^{+} \mathrm{CD}^{+}$cells in the peripheral blood did not correlate with the percentage present in the islet infiltrate $(r=0.23$, for all ages). There were also a few instances (3/30), where NRP-V7reactive $T$ cells were present in islets, but could not be detected in peripheral blood.

Dynamics of autoreactive T cells and prediction of autoimmune diabetes. Because the presence of autoreactive $T$ cells in peripheral blood reflected their accumulation in pancreatic islets, we reasoned that screening the peripheral blood of individual animals over time might allow us to predict which animals would develop diabetes. Blood was obtained weekly from 6 to 32 weeks of age, or until the onset of diabetes, and autoreactive $\mathrm{CD}^{+} \mathrm{T}$ cells were tracked using the NRP-V7 tetramer. Mice that became diabetic were maintained with exogenous insulin and monitored for four additional weeks. Several initial observations were made regarding the dynamics of circulating autoreactive $\mathrm{T}$ cells before diabetes onset (Figure 3a). The earliest detection of NRP-V7-specific T cells in blood occurred at 9 weeks of age, suggesting that before this time, there was insufficient priming and/or proliferation of this autoreactive $T$ cell population to allow visualization in blood. Since insulitis begins at approximately 3 weeks of age (25), these data also imply that 6 weeks is required for sufficient expansion of primed $\mathrm{T}$ cells in pancreatic lymph nodes for them to become visible in peripheral blood. Importantly, mice destined to develop diabetes had significantly larger populations of NRP-V7-reactive T cells in the peripheral blood before diabetes onset (i.e., between 9 and 16 weeks of age). Interestingly, these populations appeared in distinct cycles before the onset of hyperglycemia and were markedly decreased in number shortly thereafter, presumably due to lack of antigen as a result of $\beta$ cell destruction.

To determine whether the presence of $\beta$ cell-specific $T$ cells in peripheral blood could be used to predict diabetes development, the cumulative percentage of NRP-V7-specific T cells during the prediabetic period was calculated (Figure 3b). Mice that eventually developed diabetes accumulated a significantly larger proportion of NRP-V7-reactive T cells over time, evident several weeks before the average age of diabetes onset (18.5 weeks). All mice (13/13) that accumulated $0.75 \%$ NRP-V7 tetramer-positive cells by 15 weeks of age developed diabetes, whereas all of those that did not $(5 / 5)$, remained diabetes free for the entire 32-week study period (Table 2). Further analysis of the individual mouse data revealed that in the context of weekly monitoring, a single value of greater than $0.50 \%$ NRP-V7-reactive T cells in peripheral blood is a strong predictor of diabetes. For example, of the 13 mice that went on to become diabetic, 11 had at least one blood sample showing greater
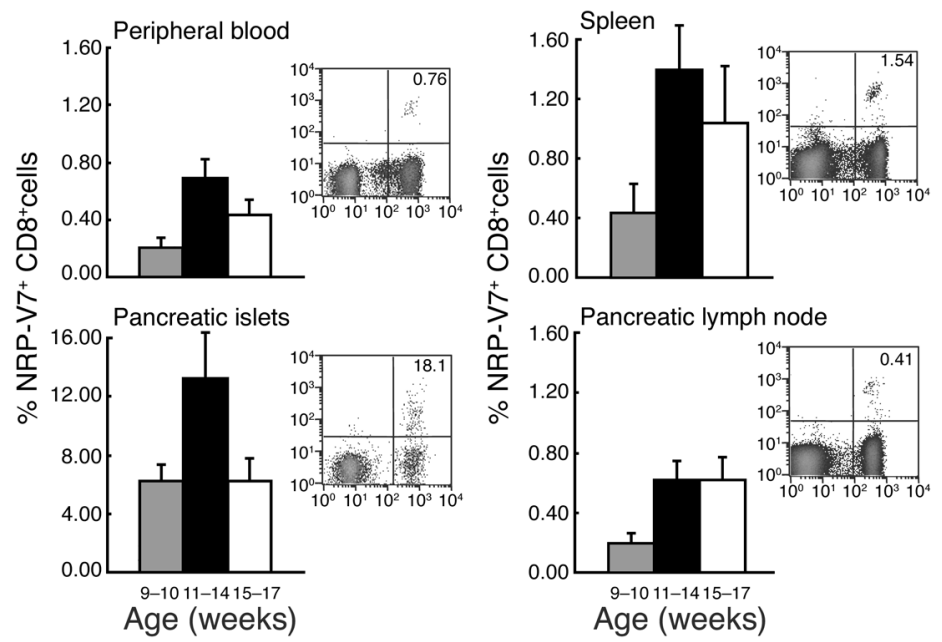

Figure 2

NRP-V7-reactive $T$ cells detected in peripheral blood indicate their presence in pancreatic islets. The mean ( \pm SEM) percentage of NRP-V7 tetramer-positive $\mathrm{CD}^{+} \mathrm{B} 220^{-}$cells within peripheral blood, pancreatic islets, spleen, and pancreatic draining lymph nodes are shown for individual NOD mice of 9-10 (gray bars; $n=7$ ), $11-14$ (black bars; $n=13$ ), and 15-17 (white bars; $n=10$ ) weeks of age. The inset shows a representative staining of each tissue from a single 12 -week-old mouse. Numbers indicate the percentage of $\mathrm{CD} 8^{+} \mathrm{B} 220^{-}$tetramerpositive cells. 
than $0.50 \%$ NRP-V7-reactive CD8 ${ }^{+} \mathrm{T}$ cells (Figure $3 \mathrm{c}$ ). In contrast, none of the five mice that failed to progress to diabetes by 32 weeks of age ever exhibited NRP-V7 staining greater than $0.50 \%$. On the other hand, the data also show that if only a single measurement of NRP-reactive cells were to be made, a criteria of greater than $0.50 \%$ $\mathrm{NRP}-\mathrm{V}^{+} \mathrm{CD}^{+}$cells would not have had predictive value since the majority of values were less than $0.50 \%$, even for animals that went to develop diabetes (Figure 3c).

\section{Discussion}

Identification and characterization of antigen-specific $T$ cells directly ex vivo during the pathogenesis of autoimmune diseases has proved elusive, in part because of the problems associated with identification of the relevant antigens, and in part because autoreactive $T$ cells are thought to be infrequent and of relatively low avidity for their target peptide. We have demonstrated previously that the islets of prediabetic NOD mice contain a significant number of $\mathrm{CD}^{+} \mathrm{T}$ cells specific for the peptide mimotope NRP (10). In those experiments, however, islet-associated $\mathrm{T}$ cells were expanded by culture with IL-2 before tetramer staining, possibly altering the true proportion of autoreactive cells present. In this study, an MHC class I tetramer complexed to a high-affinity ligand of NRP-reactive T cells (NRP-V7) was used to show that unmanipulated islet cells examined directly ex vivo also contain a significant number of functional autoreactive $\mathrm{CD}^{+} \mathrm{T}$ cells (up to $37 \%$ of all $\mathrm{CD}^{+} \mathrm{T}$ cells within islets at any given time recognize the peptide NRP-V7). We further demonstrated, we believe for the first time, that it is possible to visualize autoreactive $\mathrm{CD}^{+} \mathrm{T}$ cells in peripheral blood ex vivo prior to development of a spontaneous autoimmune disease. We found that NOD mice destined to develop diabetes had a significantly higher number of $\beta$ cell-specific T cells in the peripheral blood, and, accordingly, quantification of these cells resulted in an effective means of predicting diabetes outcome.

In general, the presence of autoreactive T cells in peripheral blood signified the presence of inflammatory cells in pancreatic islets. Although a significant correlation between the number of NRP-V7-reactive $\mathrm{CD}^{+}$ cells in the peripheral blood and pancreatic islets was not observed, this

$$
\text { C }
$$

\section{Figure 3}

was not unexpected since recently activated autoreactive $\mathrm{CD}^{+} \mathrm{T}$ cells in the peripheral blood were likely in the process of trafficking to pancreatic islets. In support of this notion, tetramer-positive autoreactive cells in the blood also expressed the $\mathrm{T}$ cell activation markers CD69 and CD44 (data not shown).

The ability to analyze the proportion of NRP-V7 tetramer-positive cells in peripheral blood on a weekly basis during the prediabetic period revealed novel information regarding circulating autoreactive $T$ cells during the development of autoimmune diabetes. Those
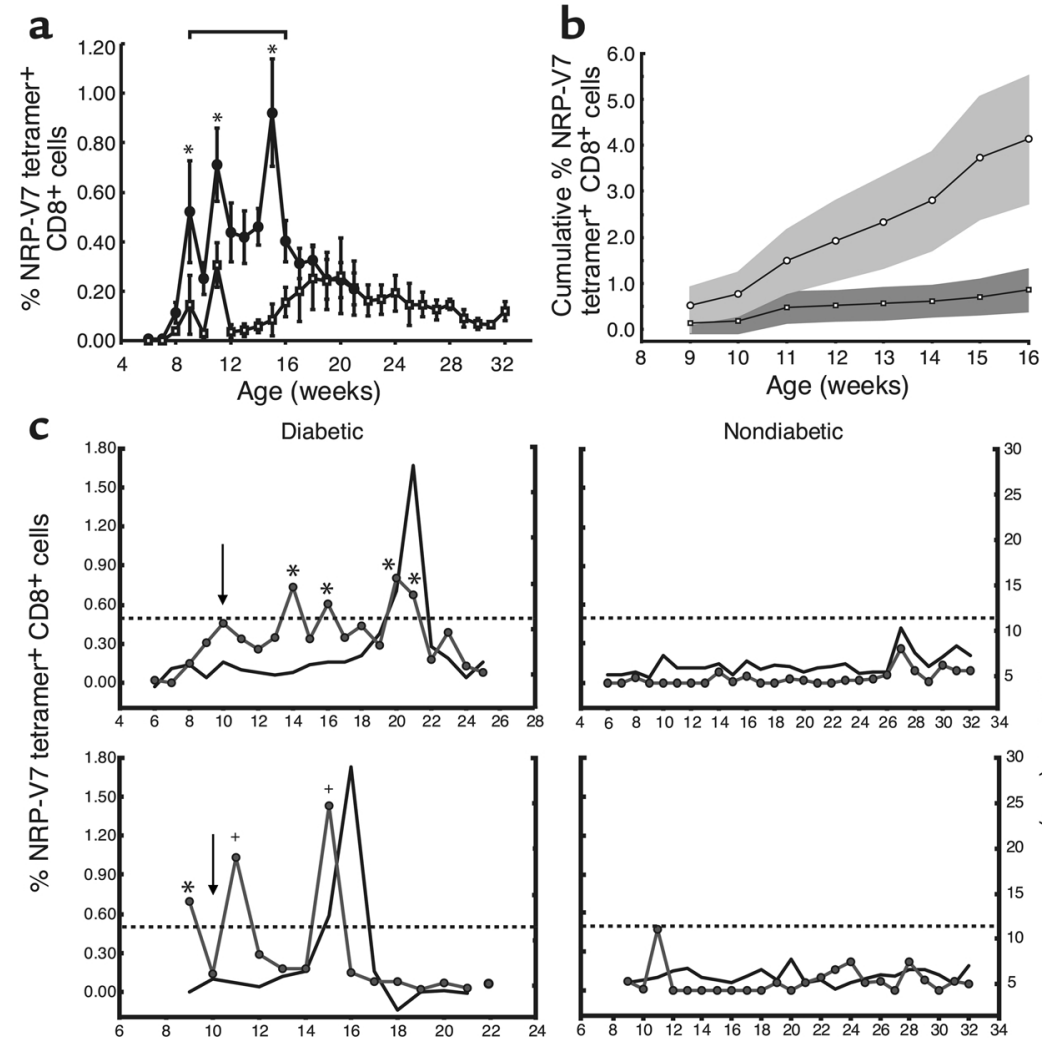

Age (weeks)

NRP-V7-specific T cells in peripheral blood can be used to predict diabetes development. (a) Mean proportion ( \pm SEM) of NRP-V7 tetramer-positive CD8 ${ }^{+}$B220- cells in peripheral blood of mice that developed diabetes (filled circles; $n=13$ ) versus mice that remained nondiabetic to 32 weeks of age (open squares; $n=5$ ). Mice from different litters were analyzed in two groups, beginning at $6(n=6)$ or 9 weeks of age $(n=12)$. Most diabetic mice were removed from the study after 21 weeks of age. The difference in NRP-V7 tetramer-positivity between diabetic and nondiabetic mice was significant for every time point from 9 to 16 weeks of age (bar; $P<0.001$ ). For diabetic animals, the differences between NRP-V7 tetramer-positive peaks $\left({ }^{*}\right)$ and the time points before and after the peak were also significant $(P<0.001)$. (b) Accumulation of NRP-V7 tetramerpositive cells in peripheral blood of diabetic (open circles; $n=13$ ) and nondiabetic (open squares; $n=5$ ) mice from 9 to 16 weeks of age. Weekly measurements of NRP-V7 tetramer-positive cells were summed cumulatively and expressed as a mean \pm the $95 \%$ confidence interval (shading). (c) Representative data from individual mice showing the proportion of NRP-V7 tetramer-positive cells in peripheral blood (filled circles) along with blood glucose (solid line, no symbols). Glucose normalization following hyperglycemia was due to insulin treatment. Arrows indicate the point at which mice had accumulated $0.75 \%$ NRP-V7 tetramer-positive cells, predictive of diabetes development. Time points where NRP-V7 tetramer staining exceeded $0.50 \%\left({ }^{*}\right)$ or $1.00 \%(+)$ are indicated along with the $0.50 \%$ threshold (dashed line). 


\section{Table 2}

Accuracy of cumulative peripheral blood NRP-V7 tetramer measurements $(0.75 \%)$ for the prediction of diabetes

\begin{tabular}{lllc}
\hline Age (weeks) & 11 & 13 & 15 \\
Sensitivity (\%) & 62 & 85 & 100 \\
Specificity (\%) & 80 & 80 & 60 \\
Positive predictive value (\%) & 89 & 92 & 87 \\
Negative predictive value (\%) & 44 & 67 & 100 \\
\cline { 1 - 2 } & & &
\end{tabular}

mice destined to develop diabetes not only had a significantly larger proportion of NRP-V7-specific CD8 ${ }^{+} \mathrm{T}$ cells in the peripheral blood, but the appearance of these autoreactive cells occurred in distinct cycles. This finding raises the possibility that each cycle represented a round of clonal proliferation of autoreactive $T$ cells undergoing avidity maturation $(10,26,27)$. In this model, the final expansion before disease onset would be the $T$ cell population with the highest $\beta$ cell avidity, capable of the most efficient $\beta$ cell destruction.

Importantly, the quantification of autoreactive $\mathrm{T}$ cells in the peripheral blood each week permitted prediction of diabetes occurrence. A cumulative sum of $0.75 \%$ NRP-V7-reactive cells in the peripheral blood between 9 and 16 weeks of age predicted diabetes outcome with increasing sensitivity as the animals approached hyperglycemia. We also observed that in the context of weekly monitoring, any mouse with greater than $0.50 \%$ NRP-V7-reactive cells at any one time became diabetic. Even for mice that went on to develop diabetes, however, the majority of their weekly samples contained less than $0.50 \%$ NRP-V7-reactive $\mathrm{CD} 8^{+}$cells (Figure 3c). Thus, for any prospective study to predict diabetes occurrence in mice, our experience suggests that weekly monitoring will be required, and that the cumulative sum of autoreactive $T$ cells (rather than a single measurement) will be the most useful and accurate predictor.

Prediction of diabetes in humans or mice is currently based on the presence of autoantibodies targeted to the $\beta$ cell proteins insulin, glutamic acid decarboxylase (GAD), and ICA512/IA-2. In humans, the presence of all three autoantibodies predicts the development of diabetes within $5-10$ years with a sensitivity ranging from $60 \%-100 \%(4,28)$. In mice, the presence of serum autoantibodies has been shown to vary considerably between both diabetic and nondiabetic mice $(5,29,30)$. A recent workshop validated the presence of antiinsulin autoantibodies (anti-IAA) in prediabetic NOD mice, but questioned the role of both ICA512/IA-2 and GAD Ab's (5). Consistent with these findings is a recent study showing that the presence of IAA in NOD mice is strongly associated with development of diabetes and that the age of IAA appearance correlates with the age of disease onset (31). Because tetramers directly detect $\beta$ cell-specific effector $T$ cells, tetramer screening offers a complementary approach for identification of prediabetic individuals. Because IAA appear first at 8 weeks of age and the presence of NRP-reactive T cells appear at 9 weeks of age, these two assays together provide a very powerful tool for diabetes prediction in the NOD mouse. While the utility of human leukocyte antigen (HLA) tetramers for predicting human disease cannot be assessed until human CTL autoepitopes are identified, we envision that they may eventually permit earlier and more specific prediction of disease. In particular, tetramers might be used to identify prediabetic individuals well before disease onset, potentially allowing for interventions aimed at preserving $\beta$ cell mass.

The ability to visualize a significant number of autoreactive $\mathrm{CD}^{+} \mathrm{T}$ cells resulted from the application of an MHC class I tetramer complexed with a high-avidity peptide analogue of a $\beta$ cell epitope. Development of high-avidity mimics of low-avidity autoepitopes thus introduces a novel general principle for the design and use of tetramers aimed at detecting autoreactive T cells. In light of these results, it is reasonable to speculate that previous attempts to detect autoreactive $\mathrm{T}$ cells in peripheral blood were hampered by the low avidity, in combination with the low frequency, of target $T$ cells. In fact, previous attempts to detect circulating GAD-specific CD4 $4^{+} \mathrm{T}$ cells $(12)$ or INS-specific $\mathrm{CD}^{+} \mathrm{T}$ cells $(9)$ in NOD mice or other naturally occurring autoepitopes in different autoimmune diseases $(11,13,14)$ using MHCtetramers required expansion of $\mathrm{T}$ cells either by repeated immunizations with the target peptide or prolonged in vitro culture. Previous work examining autoreactive $\mathrm{CD}^{+} \mathrm{T}$ cell populations in pancreatic islets taken directly ex vivo using an endogenous insulin peptide found that up to $80 \%$ of islet-associated $\mathrm{T}$ cells were specific for a peptide derived from the INS (9). In our study, we were also able to detect INS-specific $\mathrm{CD} 8^{+}$cells in the pancreatic islets of young NOD mice, albeit at a lower frequency, but we were unable to detect these cells in the peripheral blood. Because the endogenous counterpart for the NRP peptide is not yet known, we do not know how its avidity compares with the avidity of the naturally occurring peptide. Given that analogues of increasing avidity (NRP-A7 and NRP-V7) can detect a higher proportion of NRP-reactive cells, it suggests that highavidity analogues of naturally occurring epitopes might also be required to visualize low-avidity autoreactive cells. In light of these results, a high-avidity heteroclitic analogue of the insulin peptide might permit more sensitive detection of INS-reactive CTLs, particularly in young mice, thereby clarifying further the role of the INS epitope in the progression of disease.

Identification of antigen-specific $T$ cells in peripheral blood with MHC tetramers has thus far only been possible following viral (7) or bacterial (6) infection, where CTLs specific for those pathogens are thought to be of relatively high affinity for their target antigen. It is interesting that the only instance where autoreactive $T$ cells have been identified in peripheral blood using MHC class I tetramers before our study is in the case of vitiligo, a chronic autoimmune skin disease (8). In vitiligo, CTLs specific for a melanocyte antigen were found in the peripheral blood of patients, and the frequency of 
melanocyte-specific CTLs was proportional to the severity of disease. These findings support our hypothesis that autoreactive $\mathrm{T}$ cells are difficult to detect in the peripheral blood prior to symptoms of autoimmune disease because of both their low avidity and low frequency. In a disease such as vitiligo, where the target antigen in skin is maintained for a prolonged period, continual stimulation of $\mathrm{T}$ cell populations is likely to generate highly avid autoreactive $T$ cells that are easier to detect. Moreover, because autoreactive CTLs were visualized in blood obtained from patients with ongoing disease, it is not surprising that the frequency of autoreactive cells would also be high. This is not the case in autoimmune diabetes, where at the time of clinical presentation the bulk of the cognate antigen ( $\beta$ cell proteins) is lost, and the frequency of autoreactive $\mathrm{T}$ cells is low (Figure 3c).

Finally, peripheral blood measurements of autoreactive $\mathrm{CD}^{+} \mathrm{T}$ cells using tetramers may offer possibilities beyond type 1 diabetes. Our findings suggest that it will be possible to detect and quantify autoreactive $T$ cells in other autoimmune diseases that involve $\mathrm{CD}^{+} \mathrm{T}$ cells, such as experimental autoimmune encephalomyelitis or primary biliary cirrhosis (32). Indeed, a high priority should now be placed on the identification of human $\mathrm{CD}^{+} \mathrm{T}$ cell epitopes in diabetes, as well as other autoimmune disorders, to exploit HLA tetramers for prediction of disease.

\section{Acknowledgments}

We are indebted to Galina Soukatcheva and the University of British Columbia Pathology Islet Isolation Core for the provision of mouse islets. We are grateful to Gregor Reid, Barry Mason, Sarah Lasuta, Sabrina Tafuro, and Dina Panagiotopoulos for stimulating discussions and technical assistance. We thank also Sarah Townsend for thoughtful comments. J.D. Trudeau is supported by fellowships from the Canadian Institutes of Health Research/Diabetic Children's Foundation and The Michael Smith Foundation for Health Research. P. Santamaria is a Senior Scholar, and J.F. Elliott a Scientist of the Alberta Heritage Foundation for Medical Research. This work was supported by grants from The Canadian Diabetes Association in honor of the late Violet D. Mulcahy and The British Columbia's Children's Hospital Foundation (to R. Tan), and the Canadian Institutes of Health Research (CIHR) (to P. Santamaria). The authors are members of The Juvenile Diabetes Research Foundation/CIHR funded $\beta$-cell Apoptosis Network ( $\beta$-CAN).

1. Notkins, A.L., and Lernmark, A. 2001. Autoimmune type 1 diabetes: resolved and unresolved issues. J. Clin. Invest. 108:1247-1252. doi:10.1172/JCI200114257.

2. Tisch, R., and McDevitt, H. 1996. Insulin-dependent diabetes mellitus. Cell. 85:291-297.

3. Delovitch, T.L., and Singh, B. 1997. The nonobese diabetic mouse as a model of autoimmune diabetes: immune dysregulation gets the NOD. Immunity. 7:727-738.

4. Graves, P.M., and Eisenbarth, G.S. 1999. Pathogenesis, prediction and trials for the prevention of insulin-dependent (type 1) diabetes mellitus. Adv. Drug Deliv. Rev. 35:143-156.

5. Bonifacio, E., et al. 2001. International Workshop on Lessons from Animal Models for Human Type 1 Diabetes: identification of insulin but not glutamic acid decarboxylase or IA-2 as specific autoantigens of humoral autoimmunity in nonobese diabetic mice. Diabetes. 50:2451-2458

6. Kerksiek, K.M., and Pamer, E.G. 1999. T cell responses to bacterial infection. Curr. Opin. Immunol. 11:400-405.

7. Doherty, P.C., and Christensen, J.P. 2000. Accessing complexity: the dynamics of virus-specific T cell responses. Annu. Rev. Immunol. 18:561-592.

8. Ogg, G.S., Rod Dunbar, P., Romero, P., Chen, J.L., and Cerundolo, V. 1998. High frequency of skin-homing melanocyte-specific cytotoxic T lymphocytes in autoimmune vitiligo. J. Exp. Med. 188:1203-1208.

9. Wong, F.S., et al. 1999. Identification of an MHC class I-restricted autoantigen in type 1 diabetes by screening an organ-specific cDNA library. Nat. Med. 5:1026-1031.

10. Amrani, A., et al. 2000. Progression of autoimmune diabetes driven by avidity maturation of a T-cell population. Nature. 406:739-742.

11. Kotzin, B.L. 2000. Use of soluble peptide-DR4 tetramers to detect synovial $\mathrm{T}$ cells specific for cartilage antigens in patients with rheumatoid arthritis. Proc. Natl. Acad. Sci. USA. 97:291-296.

12. Liu, C.P., Jiang, K., Wu, C.H., Lee, W.H., and Lin, W.J. 2000. Detection of glutamic acid decarboxylase-activated T cells with I-Ag7 tetramers. Proc. Natl. Acad. Sci. USA. 97:14596-14601.

13. Meyer, A.L., et al. 2000. Direct enumeration of Borrelia-reactive CD4 T cells ex vivo by using MHC class II tetramers. Proc. Natl. Acad. Sci. USA. 97:11433-11438.

14. Buckner, J.H., Van, L.M., Kwok, W.W., and Tsarknaridis, L. 2002. Identification of type II collagen peptide 261-273-specific T cell clones in a patient with relapsing polychondritis. Arthritis Rheum. 46:238-244.

15. Liu, G.Y., et al. 1995. Low avidity recognition of self-antigen by $\mathrm{T}$ cells permits escape from central tolerance. Immunity. 3:407-415.

16. Bouneaud, C., Kourilsky, P., and Bousso, P. 2000. Impact of negative selection on the $\mathrm{T}$ cell repertoire reactive to a self-peptide: a large fraction of $\mathrm{T}$ cell clones escapes clonal deletion. Immunity. 13:829-840.

17. Atkinson, M.A., and Leiter, E.H. 1999. The NOD mouse model of type 1 diabetes: as good as it gets? Nat. Med. 5:601-604.

18. Serreze, D.V., Leiter, E.H., Christianson, G.J., Greiner, D., and Roopenian, D.C. 1994. Major histocompatibility complex class I-deficient NODB2mnull mice are diabetes and insulitis resistant. Diabetes. 43:505-509.

19. Wicker, L.S., et al. 1994. Beta 2-microglobulin-deficient NOD mice do not develop insulitis or diabetes. Diabetes. 43:500-504.

20. Anderson, B., Park, B.J., Verdaguer, J., Amrani, A., and Santamaria, P. 1999. Prevalent CD8 $(+)$ T cell response against one peptide/MHC complex in autoimmune diabetes. Proc. Natl. Acad. Sci. USA. 96:9311-9316.

21. Verdaguer, J., et al. 1997. Spontaneous autoimmune diabetes in monoclonal T cell nonobese diabetic mice. J. Exp. Med. 186:1663-1676.

22. Altman, J.D., et al. 1996. Phenotypic analysis of antigen-specific T lymphocytes. Science. 274:94-96.

23. Amrani, A., et al. 2001. Expansion of the antigenic repertoire of a single $\mathrm{T}$ cell receptor upon $\mathrm{T}$ cell activation. J. Immunol. 167:655-666.

24. Wallny, H.J., et al. 1992. Identification and quantification of a naturally presented peptide as recognized by cytotoxic $\mathrm{T}$ lymphocytes specific for an immunogenic tumor variant. Int. Immunol. 4:1085-1090.

25. Hoglund, P., et al. 1999. Initiation of autoimmune diabetes by developmentally regulated presentation of islet cell antigens in the pancreatic lymph nodes. J. Exp. Med. 189:331-339.

26. Busch, D.H., and Pamer, E.G. 1999. T cell affinity maturation by selective expansion during infection. J. Exp. Med. 189:701-710.

27. Savage, P.A., Boniface, J.J., and Davis, M.M. 1999. A kinetic basis for T cell receptor repertoire selection during an immune response. Immunity. 10:485-492.

28. Seissler, J., Hatziagelaki, E., and Scherbaum, W.A. 2001. Modern concepts for the prediction of type 1 diabetes. Exp. Clin. Endocrinol. Diabetes. 109(Suppl.):S304-S316.

29. Mackay, I.R., et al. 1996. Lack of autoimmune serological reactions in rodent models of insulin dependent diabetes mellitus. J. Autoimmun. 9:705-711.

30. Abiru, N., et al. 2001. Transient insulin autoantibody expression independent of development of diabetes: comparison of NOD and NOR strains. J. Autoimmun. 17:1-6.

31. Yu, L., et al. 2000. Early expression of antiinsulin autoantibodies of humans and the NOD mouse: evidence for early determination of subsequent diabetes. Proc. Natl. Acad. Sci. USA. 97:1701-1706.

32. Liblau, R.S., Wong, F.S., Mars, L.T., and Santamaria, P. 2002. Autoreactive CD8 $\mathrm{T}$ cells in organ-specific autoimmunity. Emerging targets for therapeutic intervention. Immunity. 17:1-6. 